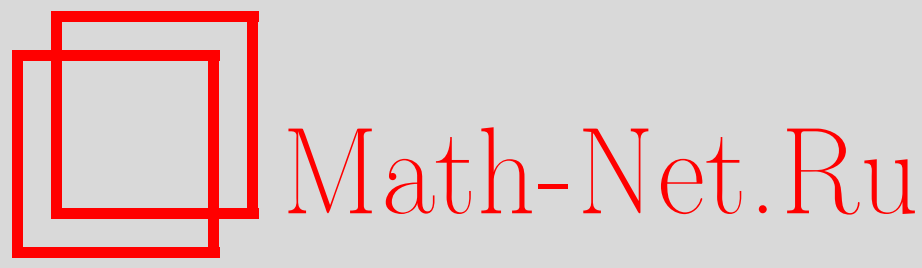

А. В. Корибут, Ковариантные структурные константы для алгебры деформированных осцилляторов, $Т M \Phi$, 2017, том 193, номер 1, 3-14

DOI: https://doi.org/10.4213/tmf9170

Использование Общероссийского математического портала Math-Net.Ru подразумевает, что вы прочитали и согласны с пользовательским соглашением http://www. mathnet.ru/rus/agreement

Параметры загрузки:

IP : 54.237 .206 .68

26 апреля 2023 г., 17:33:11

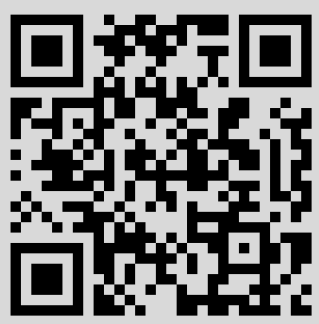




\section{КОВАРИАНТНЫЕ СТРУКТУРНЫЕ КОНСТАНТЫ ДЛЯ АЛГЕБРЫ ДЕФОРМИРОВАННЫХ ОСЦИЛЛЯТОРОВ}

Получены $s l_{2}$-ковариантные выражения для структурных констант алгебры деформированных осцилляторов $A q(2, \nu)$.

Ключевые слова: структурные константы, деформированные осцилляторы, алгебра высших спинов, гипергеометрические функции.

DOI: https://doi.org/10.4213/tmf9170

\section{1. ВВЕДЕНИЕ}

Алгебра деформированных осцилляторов, порожденная элементами $y_{\alpha}(\alpha=+,-)$ и $\mathcal{K}$, которые подчиняются соотношениям ${ }^{1)}$

$$
\left[y_{\alpha}, y_{\beta}\right]=2 i \epsilon_{\alpha \beta}(1+\nu \mathcal{K}), \quad\left\{y_{\alpha}, \mathcal{K}\right\}=0, \quad \mathcal{K}^{2}=1
$$

где $\nu \in \mathbb{C}$ - произвольный параметр, $\epsilon_{\alpha \beta}$ - полностью антисимметричный тензор с $\epsilon_{+-}=1, \mathcal{K}$ - так называемый оператор Клейна, была впервые рассмотрена Вигнером [1] в ее конкретной реализации. В работах [2] и [3] было высказано предположение, что она может быть интерпретирована как алгебра высших спинов (ВС) в $2+1$ измерениях. В немного иной реализации эта же алгебра ВС была рассмотрена в работе [4]. Кроме того, алгебра деформированных осцилляторов определяет вид нелинейных уравнений в произвольном числе измерений [5], [6]. Также эта алгебра появляется в контексте двумерных конформных теорий поля [7]-[9]. В последнее время интерес к ней повысился в контексте $\mathrm{AdS}_{3} / \mathrm{CFT}_{2}$-соответствия [10]. Анализ этой задачи затруднен, в частности, из-за отсутствия удобного выражения для произведения в этой алгебре.

Автор выражает благодарность фонду Династия за финансовую поддержку. Исследование выполнено при поддержке РФФИ (грант № 14-02-01172).

1) $\left[y_{\alpha}, y_{\beta}\right] \equiv y_{\alpha} y_{\beta}-y_{\alpha} y_{\beta},\left\{y_{\alpha}, \mathcal{K}\right\} \equiv y_{\alpha} \mathcal{K}+\mathcal{K} y_{\alpha}$.

* Московский физико-технический институт (государственный университет), Долгопрудный, Московская обл., Россия. E-mail: akoribut@gmail.com 
Рассмотрим универсальную обертывающую алгебру $s l_{2}$, она содержит идеал $\mathcal{I}_{h s}(\lambda)$, порожденный центральным элементом

$$
C-\frac{1}{4}\left(\lambda^{2}-1\right) \mathbf{1}
$$

где $C$ - квадратичный оператор Казимира алгебры $s l_{2}$, а $\mathbf{1}$ - единичный элемент в обертывающей алгебре $U\left(s l_{2}\right)$. Бозонная алгебра Ли ВС в $2+1$ измерениях - это фактор-алгебра по этому идеалу

$$
h s[\lambda]=U\left(s l_{2}\right) / \mathcal{I}_{h s}(\lambda) .
$$

Аналогично можно определить супералгебру Ли ВС, но теперь следует рассматривать обертывающую алгебру от алгебры $\operatorname{spp}(1 \mid 2)$. Она содержит идеал $\mathcal{I}_{s h s}(\lambda)$, порожденный центральным элементом

$$
C-\frac{1}{4} \lambda(\lambda-1) \mathbf{1}
$$

где $C$ - квадратичный оператор Казимира в алгебре $\operatorname{sp}(1 \mid 2)$. Фактор-алгеброй по этому идеалу называется супералгебра Ли ВС

$$
\operatorname{shs}[\lambda]=U(\operatorname{osp}(1 \mid 2)) / \mathcal{I}_{\text {shs }}(\lambda) .
$$

Структурные константы для $h s[\lambda]$ и $s h s[\lambda]$ были найдены в работах [7] и [11] соответственно.

Конкретное представление $h s[\lambda]$ можно построить, используя осцилляторы, которые подчиняются соотношениям (1) с $\nu=1-2 \lambda$. Рассмотрим следующие билинейные комбинации:

$$
J_{ \pm}=\frac{i}{4}\left(y_{ \pm}\right)^{2}, \quad J_{0}=\frac{i}{4}\left\{y_{+}, y_{-}\right\} .
$$

Можно проверить, что $\left\{J_{-}, J_{+}, J_{0}\right\}$ подчиняются коммутационным соотношениям алгебры $s l_{2}$

$$
\left[J_{+}, J_{-}\right]=2 J_{0}, \quad\left[J_{ \pm}, J_{0}\right]= \pm J_{ \pm} .
$$

Значение оператора Казимира в этом представлении совпадает с его значением в определении $h s[\lambda](3)$.

B работе [7] было введено Lone-Star-произведение как ассоциативное произведение в $h s[\lambda]$. Недавно эта задача была рассмотрена в работе [12]. Произведение было дано для функций

$$
\begin{aligned}
& L(\xi)=\sum_{n=0}^{\infty} \frac{1}{n !} \xi^{\alpha_{1} \tilde{\alpha}_{1}} \ldots \xi^{\alpha_{n} \tilde{\alpha}_{n}} y_{\left(\alpha_{1}\right.} y_{\tilde{\alpha}_{1}} \ldots y_{\alpha_{n}} y_{\left.\tilde{\alpha}_{n}\right)} \\
& L(\eta)=\sum_{n=0}^{\infty} \frac{1}{n !} \eta^{\alpha_{1} \tilde{\alpha}_{1}} \ldots \eta^{\alpha_{n} \tilde{\alpha}_{n}} y_{\left(\alpha_{1}\right.} y_{\tilde{\alpha}_{1}} \ldots y_{\alpha_{n}} y_{\left.\tilde{\alpha}_{n}\right)}
\end{aligned}
$$

где $\xi^{\alpha_{1} \tilde{\alpha}_{1}}$ и $\eta^{\alpha_{1} \tilde{\alpha}_{1}}$ - симметричные $s l_{2}$-тензоры, а $y_{\alpha}$ - осцилляторы, подчиняющиеся соотношениям (1). Также мы будем использовать следующие обозначения для полной симметризации индексов:

$$
y_{\left(\alpha_{1}\right.} y_{\alpha_{2}} \ldots y_{\left.\alpha_{n}\right)}=\frac{1}{n !}\left(y_{\alpha_{1}} y_{\alpha_{2}} \ldots y_{\alpha_{n}}+\text { перестановки }\right) .
$$


Произведение, определенное в (8), имеет следующий вид:

$$
\begin{aligned}
L(\xi) * L(\eta)= & \sum_{n=0}^{\infty}{ }_{2} F_{1}\left[\begin{array}{rr}
n+\frac{3-\nu}{2} & n+\frac{1+\nu}{2} \\
n+\frac{3}{2}
\end{array} ;-\frac{1}{4} \phi\right] \times \\
& \times \frac{1}{n !} \zeta^{\alpha_{1} \tilde{\alpha}_{1}} \ldots \zeta^{\alpha_{n} \tilde{\alpha}_{n}} y_{\left(\alpha_{1}\right.} y_{\tilde{\alpha}_{1}} \ldots y_{\alpha_{n}} y_{\left.\tilde{\alpha}_{n}\right)},
\end{aligned}
$$

где $\zeta^{\alpha \beta}=\xi^{\alpha \beta}+\eta^{\alpha \beta}+\xi^{\alpha \gamma} \eta^{\delta \beta} \epsilon_{\gamma \delta}$ и $\phi=\xi^{\alpha_{1} \alpha_{2}} \eta^{\beta_{1} \beta_{2}} \epsilon_{\alpha_{1} \beta_{1}} \epsilon_{\alpha_{2} \beta_{2}}$. Так как в разложениях (8) и (10) встречаются только четные степени, то оператор Клейна можно рассматривать как число.

В алгебре ВС в высших измерениях есть звездочное произведение

$$
(f \star g)(y)=\frac{1}{(2 \pi)^{2 p}} \int d^{2 p} u d^{2 p} v e^{i u_{\mu} v^{\mu}} f(y+u) g(y+v),
$$

где $f$ и $g$ - функции от коммутирующих переменных $y_{\mu}, \mu=1, \ldots, 2 p$. Формула (11) определяет ассоциативное произведение с соотношением

$$
y_{\mu} \star y_{\nu}-y_{\nu} \star y_{\mu}=2 i C_{\mu \nu}
$$

где $C_{\mu \nu}$ - симплектическая форма. Индексы поднимаются и опускаются следующим образом:

$$
y^{\mu}=C^{\mu \nu} y_{\nu}, \quad y_{\mu}=y^{\nu} C_{\nu \mu} .
$$

Не известно аналогов произведения (11) для случая деформированных осцилляторов. Но был сделан шаг вперед в вычислении звездочного произведения. Исходные и конечные функции являются рядами по степеням осцилляторов, свернутых с полностью симметричными тензорами (вейлевское упорядочение):

$$
f(y)=\sum_{n=0}^{\infty} f^{\alpha(n)} \underbrace{y_{\alpha} \ldots y_{\alpha}}_{n}, \quad f^{\alpha(n)}=f^{\left(\alpha_{1} \ldots \alpha_{n}\right)},
$$

где $f^{\alpha(n)}-$ полностью симметричный $s l_{2}$-тензор ранга $n$,

$$
\begin{aligned}
f^{\alpha(m)} & \underbrace{y_{\alpha} \ldots y_{\alpha}}_{m} * g^{\beta(n)} \underbrace{y_{\beta} \ldots y_{\beta}}_{n}= \\
& =f^{\alpha(m)} g^{\beta(n)} \sum_{p=0}^{\min (m, n)}\left(\epsilon_{\alpha \beta}\right)^{p} \underbrace{\overbrace{y_{\alpha} \ldots y_{\alpha}}^{m-p} \overbrace{y_{\beta} \ldots y_{\beta}}^{n-p}}_{m+n-2 p} \widetilde{A}(m, n, p, \nu \mathcal{K}) .
\end{aligned}
$$

Осцилляторы полностью симметризованы. Выражения для $\widetilde{A}$ в различных случаях получены в настоящей работе. Ассоциативная алгебра с соотношением (15) называется $A q(2, \nu)$. Ассоциированная с ней алгебра Ли со скобкой Ли в виде градуированного коммутатора является супералгеброй Ли ВС $\operatorname{sh} s[\lambda]$ со следующей $\mathbb{Z}_{2}$-градуировкой:

$$
\mathcal{K} f^{\alpha(n)} \underbrace{y_{\alpha} \ldots y_{\alpha}}_{n}=(-1)^{n} f^{\alpha(n)} \underbrace{y_{\alpha} \ldots y_{\alpha}}_{n} \mathcal{K} .
$$


Рассматривая только четные степени в разложении (14) и скобку Ли в виде обычного коммутатора, получим $h s[\lambda]$.

Статья организована следующим образом: в разделе 2 даны структурные константы и ковариантное выражение для произведения в алгебре $A q(2, \nu)$, для доказательства верности выражений в разделе 3 выводится условие ассоциативности, которое проверяется в приложении, в разделах 4 и 5 выводятся структурные константы для недостающих случаев.

\section{2. СТРУКТУРНЫЕ КОНСТАНТЫ ДЛЯ АССОЦИАТИВНОЙ АЛГЕБРЫ $A q(2, \nu)$}

В силу (1) оператор Клейна появляется только в комбинации $\nu \mathcal{K}$, поэтому для краткости обозначим $\hat{\nu}=\nu \mathcal{K}$. Общее выражение для $\widetilde{A}(m, n, p, \hat{\nu})$ из $(15)$ довольно громоздкое, поэтому удобно рассматривать различные случаи. Выпишем окончательные результаты для структурных констант $A q(2, \nu)$ :

1) в четно-четном случае

$$
\begin{aligned}
& A(m, n, p, \hat{\nu})= \\
& \quad=i^{p} \frac{m ! n !}{(m-p) !(n-p) ! p !}{ }_{4} F_{3}\left[\begin{array}{cccc}
1-\frac{\hat{\nu}}{2} & \frac{\hat{\nu}}{2} & \frac{-p}{2} & \frac{1-p}{2} \\
\frac{1-m}{2} & \frac{1-n}{2} & \frac{m+n-2 p+3}{2} &
\end{array}\right],
\end{aligned}
$$

2) в нечетно-нечетном случае

$$
\begin{aligned}
B(m+ & 1, n+1, p, \hat{\nu})=A(m, n, p,-\hat{\nu})+i(m+n-2 p+3+\hat{\nu}) A(m, n, p-1,-\hat{\nu})+ \\
+ & i^{2}(m-p+2)(n-p+2) \times \\
& \times \frac{m+n-2 p+5+\hat{\nu}}{m+n-2 p+5} \frac{m+n-2 p+3+\hat{\nu}}{m+n-2 p+3} A(m, n, p-2,-\hat{\nu}),
\end{aligned}
$$

3) в четно-нечетном случае

$$
\begin{aligned}
& C(m, n+1, p, \hat{\nu})= \\
& \quad=A(m, n, p,-\hat{\nu})+i(m-p+1) \frac{m+n-2 p+3+\hat{\nu}}{m+n-2 p+3} A(m, n, p-1,-\hat{\nu}),
\end{aligned}
$$

4) в нечетно-четном случае

$$
D(m+1, n, p, \hat{\nu})=A(m, n, p, \hat{\nu})+i(n-p+1) \frac{m+n-2 p+3-\hat{\nu}}{m+n-2 p+3} A(m, n, p-1, \hat{\nu}),
$$

где $m, n$ - четные числа. Обобщенные гипергеометрические функции из правой части (17) определяются следующим образом:

$$
{ }_{4} F_{3}\left[\begin{array}{llll}
a & b & c & d \\
e & f & g & ; z
\end{array}\right]=\sum_{q=0}^{\infty} \frac{(a)_{q}(b)_{q}(c)_{q}(d)_{q}}{(e)_{q}(f)_{q}(g)_{q}} \frac{z^{q}}{q !},
$$

где $(a)_{q} \equiv \Gamma(a+q) / \Gamma(a)-$ нисходящий символ Похгаммера.

Для произвольных формальных рядов

$$
f(y)=\sum_{m=0}^{\infty} f^{\alpha(m)} \underbrace{y_{\alpha} \ldots y_{\alpha}}_{m}, \quad g(y)=\sum_{n=0}^{\infty} g^{\beta(n)} \underbrace{y_{\beta} \ldots y_{\beta}}_{n}
$$


произведение имеет вид

$$
\begin{aligned}
& f(y) * g(y)=\sum_{k=0}^{\infty} \sum_{p=0}^{\infty}(f^{\alpha(2 k)} g^{\beta(2 p)} \sum_{l=0}^{\min (2 k, 2 p)}\left(\epsilon_{\alpha \beta}\right)^{l} \underbrace{\overbrace{y_{\alpha} \ldots y_{\alpha}}^{2 k-l} \overbrace{y_{\beta} \ldots y_{\beta}}^{2 p-l}}_{2 k+2 p-2 l} A(2 k, 2 p, l, \hat{\nu})+ \\
& +f^{\alpha(2 k+1)} g^{\beta(2 p+1)} \sum_{l=0}^{\min (2 k+1,2 p+1)}\left(\epsilon_{\alpha \beta}\right)^{l} \underbrace{\overbrace{y_{\alpha} \ldots y_{\alpha}}^{2 k+1-l} \overbrace{y_{\beta} \ldots y_{\beta}}^{2 p+1-l}}_{2 k+2 p+2-2 l} B(2 k+1,2 p+1, l, \hat{\nu})+ \\
& +f^{\alpha(2 k)} g^{\beta(2 p+1)} \sum_{l=0}^{\min (2 k, 2 p+1)}\left(\epsilon_{\alpha \beta}\right)^{l} \underbrace{y_{\alpha}}_{\underbrace{2 k-l}_{2 k+2 p+1-2 l} \overbrace{y_{\alpha}} \overbrace{y_{\beta} \ldots y_{\beta}}^{2 p+1-l}} C(2 k, 2 p+1, l, \hat{\nu})+ \\
& +f^{\alpha(2 k+1)} g^{\beta(2 p)} \sum_{l=0}^{\min (2 k+1,2 p)}\left(\epsilon_{\alpha \beta}\right)^{l} \underbrace{\overbrace{y_{\alpha} \ldots y_{\alpha}}^{2 k+1-l} \overbrace{y_{\beta} \ldots y_{\beta}}^{2 p-l}}_{2 k+2 p+1-2 l} D(2 k+1,2 p, l, \hat{\nu})) .
\end{aligned}
$$

Верность выражений (17)-(20) доказывается в следующих разделах с использованием ассоциативности алгебры $A q(2, \nu)$.

\section{3. УСЛОВИЕ АССОЦИАТИВНОСТИ}

В этом разделе выводится условие ассоциативности на бозонные структурные константы, т. е. в четно-четном случае. Для вывода других структурных констант (не в четно-четном случае) используются бозонные константы и ассоциативность алгебры, поэтому достаточно рассмотреть только этот случай. Произведение двух мономов может быть записано в виде

$$
\begin{aligned}
f^{\alpha(m)} & \overbrace{y_{(\alpha} \ldots y_{\alpha)}}^{m} * g^{\beta(n)} \overbrace{y_{(\beta} \ldots y_{\beta)}}^{n}= \\
& =f^{\alpha(m)} g^{\beta(n)} \sum_{p=0}^{\min (m, n)}\left(\epsilon_{\alpha \beta}\right) \underbrace{p}_{m+n-2 p} \underbrace{n-p}_{\underbrace{m-p}_{y_{\left(\alpha \ldots y_{\alpha}\right.}} \overbrace{\left.y_{\beta} \ldots y_{\beta}\right)}^{n-p}} A(m, n, p, \hat{\nu}),
\end{aligned}
$$

где $A(m, n, p, \hat{\nu})$ - структурные константы для четно-четного случая. В этом разделе мы рассматриваем только четные мономы. Можно переписать предыдущее уравнение в немного другой форме:

$$
\begin{aligned}
f^{\alpha(m)} \overbrace{y_{\left(\alpha \cdots y_{\alpha)}\right.}}^{m} * g^{\beta(n-2) \gamma_{1} \gamma_{2}} \overbrace{\overbrace{\left(\beta \ldots y_{\beta}\right)}}^{n-2} * y_{\gamma_{1}} y_{\gamma_{2}}= \\
=f^{\alpha(m)} g^{\beta(n-2) \gamma_{1} \gamma_{2}} \times \\
\quad \times(\sum_{p=0}^{\min (m, n-2)}\left(\epsilon_{\alpha \beta}\right)^{p} \underbrace{\overbrace{y_{\left(\alpha \ldots y_{\alpha}\right.}}^{m-p} \overbrace{\left.y_{\beta} \ldots y_{\beta}\right)}^{n-2-p}}_{m+n-2-2 p} A(m, n-2, p, \hat{\nu})) * y_{\gamma_{1}} y_{\gamma_{2}} .
\end{aligned}
$$

Здесь использовалось свойство ассоциативности произведения. Можно умножить $m \times n$ непосредственно или $m \times(n-2) \times 2$. В силу ассоциативности результат должен быть тем же. Чтобы выразить это условие в форме уравнения, нужно 
симметризовать осцилляторы с индексами $\gamma_{1}$ и $\gamma_{2}$. Остальные осцилляторы уже симметризованы:

$$
\begin{aligned}
& \underbrace{m-2-p}_{\underbrace{y_{(\alpha+2-2 p}}_{\left(\alpha \ldots y_{\alpha}\right.} \overbrace{\left.y_{\beta} \ldots y_{\beta}\right)}^{n-p}} y_{\gamma_{1}} y_{\gamma_{2}}=[\overbrace{y_{(\alpha} \ldots y_{\alpha}}^{m-p} \overbrace{y_{\beta} \ldots y_{\beta}}^{n-2-p} y_{\left.\gamma_{1}\right)}+ \\
& \quad+\frac{2 i}{2} \frac{(m+n-2 p-2)}{m+n-1-2 p} \xi \epsilon_{\alpha \gamma_{1}} \overbrace{y_{(\alpha} \ldots y_{\alpha}}^{m-1-p} \overbrace{\left.y_{\beta} \ldots y_{\beta}\right)}^{n-2-p}(m+n-2 p-1-\hat{\nu})] y_{\gamma_{2}} .
\end{aligned}
$$

Множитель

$$
\frac{2 i}{2} \frac{(m+n-2 p-2)}{m+n-1-2 p} \xi(m+n-2 p-1-\hat{\nu})
$$

который появляется во втором слагаемом, содержащем $\epsilon_{\alpha \gamma_{1}}$, состоит из двух частей. Первая часть

$$
\frac{2 i}{2} \frac{(m+n-2 p-2)}{m+n-1-2 p}(m+n-2 p-1-\hat{\nu})
$$

есть результат симметризации $m+n-2 p-2$ осцилляторов с еще одним осциллятором:

$$
\begin{aligned}
& \underbrace{y_{\left(\rho \ldots y_{\rho)}\right.}}_{m+n-2-2 p} y_{\gamma_{1}} y_{\gamma_{2}}=[\underbrace{y_{\left(\rho \ldots y_{\rho}\right.}}_{m+n-2-2 p} y_{\left.\gamma_{1}\right)}+ \\
& \quad+\frac{2 i}{2} \frac{(m+n-2 p-2)}{m+n-1-2 p} \underbrace{y_{\left(\rho \cdots y_{\rho)}\right.}}_{m+n-3-2 p} \epsilon_{\rho \gamma_{1}}(m+n-2 p-1-\hat{\nu})] y_{\gamma_{2}} .
\end{aligned}
$$

Вторая часть, собственно $\xi$, чисто комбинаторная. Она появляется из-за того, что $y_{\beta}$ и $y_{\gamma_{1}}$ уже симметризованы. Чтобы получить значение $\xi$, следует найти симметрии исходного выражения

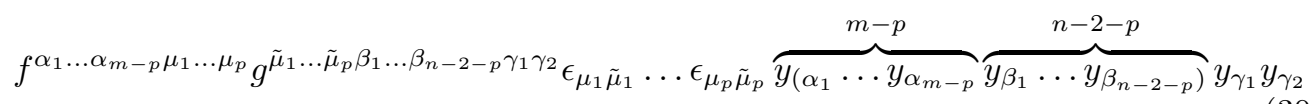

и свертки

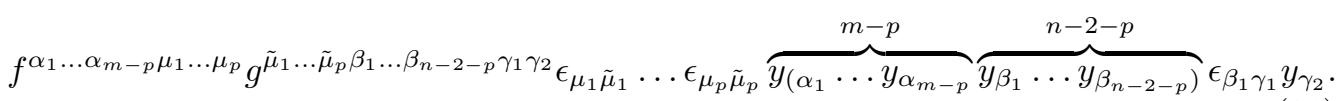

Количество перестановок, которые дают нуль, составляет

$$
(n-2-p) \frac{(m+n-2 p-3) !}{(m-p) !},
$$

а количество перестановок, которые дают ненулевые свертки, -

$$
\begin{aligned}
\xi & =\frac{(m-p) !}{(m+n-2 p-2) !}\left[\frac{(m+n-2 p-2) !}{(m-p) !}-(n-2-p) \frac{(m+n-2 p-3) !}{(m-p) !}\right]= \\
& =\frac{m-p}{m+n-2 p-2} .
\end{aligned}
$$


Необходимо повторить эту же операцию с оставшимся осциллятором $y_{\gamma_{2}}$. С учетом (25) это дает

$$
\begin{aligned}
& f^{\alpha(m)} g^{\beta(n)} \sum_{p=0}^{\min (m, n-2)} A(m, n-2, p, \hat{\nu})\left(\epsilon_{\alpha \beta}\right)^{p}[\overbrace{y_{(\alpha} \ldots y_{\alpha}}^{m-p} \overbrace{\left.y_{\beta} \ldots y_{\beta}\right)}^{n-p}+ \\
& +(2 i)(m-p) \epsilon_{\alpha \beta} \overbrace{y_{(\alpha} \ldots y_{\alpha}}^{m-p-1} \overbrace{\left.y_{\beta} \ldots y_{\beta}\right)}^{n-p-1}+ \\
& +\frac{(2 i)^{2}}{4}\left(\epsilon_{\alpha \beta}\right)^{2}(m-p)(m-p-1) \times \\
& \times \frac{m+n-2 p-1-\hat{\nu}}{m+n-2 p-1} \frac{m+n-2 p-3+\hat{\nu}}{m+n-2 p-3} \overbrace{y_{(\alpha} \ldots y_{\alpha}}^{m-p-2} \overbrace{\left.y_{\beta} \ldots y_{\beta}\right)}^{n-p-2}], \\
& A(m, n, 0, \hat{\nu})=A(m, n-2,0, \hat{\nu}), \\
& A(m, n, 1, \hat{\nu})=A(m, n-2,1, \hat{\nu})+2 i m A(m, n-2,0, \hat{\nu}), \\
& A(m, n, p, \hat{\nu})=A(m, n-2, p, \hat{\nu})+2 i(m-p+1) A(m, n-2, p-1, \hat{\nu})+ \\
& +i^{2} A(m, n-2, p-2, \hat{\nu})(m-p+2)(m-p+1) \times \\
& \times \frac{m+n-2 p+3-\hat{\nu}}{m+n-2 p+3} \frac{m+n-2 p+1+\hat{\nu}}{m+n-2 p+1},
\end{aligned}
$$

где $p=2, \ldots, n-2$,

$$
\begin{aligned}
A(m, n, n-1, \hat{\nu})=2 i( & m-n+2) A(m, n-2, n-2, \hat{\nu})+ \\
+ & i^{2} A(m, n-2, n-3, \hat{\nu})(m-n+3)(m-n+2) \times \\
\times & \frac{m-n+5-\hat{\nu}}{m-n+5} \frac{m+3+\hat{\nu}}{m-n+3}, \\
A(m, n, n, \hat{\nu})=i^{2} A( & m, n-2, n-2, \hat{\nu})(m-n+2)(m-n+1) \times \\
\times & \frac{m-n+3-\hat{\nu}}{m-n+3} \frac{m-n+1+\hat{\nu}}{m-n+1} .
\end{aligned}
$$

Вместе со структурными константами $A(m, 2, p, \hat{\nu})$ уравнения (35)-(39) единственным образом определяют структурные константы для четно-четного случая в терминах $A(m, 2, p, \hat{\nu})$. Структурные константы для $A(m, 2, p, \hat{\nu})$ могут быть получены путем прямой симметризации, процедура почти такая же, как и при выводе условия ассоциативности. В приложении проверено, что структурные константы (17) действительно являются решением уравнений (35)-(39).

\section{4. НЕЧЕТНО-НЕЧЕТНЫЙ СЛУЧАЙ}

Чтобы вывести структурные константы для нечетных мономов, достаточно использовать ассоциативность и структурные константы для бозонного случая (четно-четный случай):

$$
\begin{aligned}
f * g & =f^{\alpha(m+1)} \overbrace{y_{\alpha} \ldots y_{\alpha}}^{m+1} * g^{\beta(n+1)} \overbrace{y_{\beta} \ldots y_{\beta}}^{n+1}= \\
& =f^{\alpha(m+1)} g^{\beta(n+1)} \sum_{p=0}^{\min (m, n)+1}\left(\epsilon_{\alpha \beta}\right) \underbrace{n+p}_{\underbrace{p}_{m+n-2 p+2} \underbrace{m+1-p}_{y_{\alpha} \ldots y_{\alpha}} \overbrace{y_{\beta} \ldots y_{\beta}}^{n+1-p}}(m+1, n+1, p, \hat{\nu}),
\end{aligned}
$$


где $m, n$ - четные числа,

$$
\begin{aligned}
& f^{\alpha(m+1)} \overbrace{y_{\alpha} \ldots y_{\alpha}}^{m+1} * g^{\beta(n+1)} \overbrace{y_{\beta} \ldots y_{\beta}}^{n+1}=f^{\alpha \alpha(m)} g^{\beta(n) \beta} y_{\alpha} * \overbrace{y_{\alpha} \ldots y_{\alpha}}^{m} * \overbrace{y_{\beta} \ldots y_{\beta}}^{m} * y_{\beta}= \\
& =f^{\alpha \alpha(m)} g^{\beta(n) \beta} y_{\alpha} * \sum_{p=0}^{\min (m, n)}\left(\epsilon_{\alpha \beta}\right)^{p} \underbrace{\overbrace{y_{\alpha} \ldots y_{\alpha}}^{m-p} \overbrace{y_{\beta} \ldots y_{\beta}}^{n-p}}_{m+n-2 p} * y_{\beta} A(m, n, p,-\hat{\nu}) .
\end{aligned}
$$

Теперь нужно выполнить симметризацию, подставляя соответствующие правильные комбинаторные факторы. Процедура такая же, как и при выводе уравнений (35)-(39), поэтому приводим только окончательный ответ:

$$
\begin{aligned}
f * g=f^{\alpha(m+1)} g^{\beta(n+1)} \sum_{p=0}^{\min (m, n)}\left(\left(\epsilon_{\alpha \beta}\right)^{p} y_{\alpha(m+1-p)} y_{\beta(n+1-p)}+\right. \\
+i\left(\epsilon_{\alpha \beta}\right)^{p+1}(m+n-2 p+1+\nu) y_{\alpha(m-p)} y_{\beta(n-p)}+ \\
+i^{2}\left(\epsilon_{\alpha \beta}\right)^{p+2}(m-p)(n-p) \frac{m+n-2 p+1+\nu}{m+n-2 p+1} \frac{m+n-2 p-1+\nu}{m+n-2 p-1} \times \\
\left.\quad \times y_{\alpha(m-1-p)} y_{\beta(n-1-p)}\right) A(m, n, p,-\hat{\nu}) .
\end{aligned}
$$

Сдвигая индексы в суммах, можно привести его к виду (40), где $B(m+1, n+1, p, \hat{\nu})$ в терминах бозонных структурных констант имеет вид

$$
\begin{aligned}
B(m+1, n+1, p, \hat{\nu})=A(m, n, p,-\hat{\nu})+i(m+n-2 p+3+\hat{\nu}) A(m, n, p-1,-\hat{\nu})+ \\
+i^{2}(m-p+2)(n-p+2) \frac{m+n-2 p+5+\hat{\nu}}{m+n-2 p+5} \times \\
\times \frac{m+n-2 p+3+\hat{\nu}}{m+n-2 p+3} A(m, n, p-2,-\hat{\nu}) .
\end{aligned}
$$

\section{5. ЧЕТНО-НЕЧЕТНЫЙ И НЕЧЕТНО-ЧЕТНЫЙ СЛУЧАИ}

Так же, как и в предыдущем разделе, можно вывести структурные константы для четно-нечетного и нечетно-четного случаев. После некоторых вычислений получаем окончательный ответ:

1) в четно-нечетном случае

$$
\begin{aligned}
& C(m, n+1, p, \hat{\nu})= \\
& \quad=A(m, n, p,-\hat{\nu})+i(m-p+1) \frac{m+n-2 p+3+\hat{\nu}}{m+n-2 p+3} A(m, n, p-1,-\hat{\nu}),
\end{aligned}
$$

2) в нечетно-четном случае

$$
D(m+1, n, p, \hat{\nu})=A(m, n, p, \hat{\nu})+i(n-p+1) \frac{m+n-2 p+3-\hat{\nu}}{m+n-2 p+3} A(m, n, p-1, \hat{\nu})
$$




\section{6. ВЫВОД}

Все структурные константы для алгебры $A q(2, \nu)$ в ковариантном базисе получены. Ассоциативность произведения доказана. Тот факт, что структурные константы являются заальшутсовскими гипергеометрическими функциями, существен. Преобразование Заальшутса является ключевым свойством для доказательства ассоциативности.

\section{Проверка ассоциативности}

\section{ПРИЛОЖЕНИЕ}

Ассоциативное произведение в работе [7] было дано в так называемом конформном базисе:

$$
V_{m}^{s-1} \equiv(-1)^{s-m} \frac{(s+m) !}{(2 s) !} \underbrace{\left[J_{-}, \ldots\left[J_{-},\left[J_{-}\right.\right.\right.}_{s-m} J_{+}^{s}]]] .
$$

Подставляя в предыдущее выражение явную реализацию генераторов алгебры $s l_{2}$, можно убедиться, что

$$
V_{m}^{s-1} \sim \underbrace{y_{\left(+\cdots y_{+}\right.} \overbrace{y_{-} \cdots y_{-)}}^{s-m}}_{2 s} .
$$

Lone-Star-произведение определяется следующим образом:

$$
V_{m}^{s} * V_{n}^{t}=\frac{1}{2} \sum_{u=1}^{s+t-1} g_{u}^{s t}(m, n, \lambda) V_{m+n}^{s+t-u}
$$

где

$$
\begin{aligned}
& g_{u}^{s t}(m, n, \lambda)=\frac{(1 / 4)^{u-2}}{2(u-1) !}{ }_{4} F_{3}\left[\begin{array}{cccc}
\frac{1}{2}+\lambda & \frac{1}{2}-\lambda & \frac{2-u}{2} & \frac{1-u}{2} \\
\frac{3-2 s}{2} & \frac{3-2 t}{2} & \frac{1}{2}+s+t-u &
\end{array}\right] \times \\
& \times \sum_{k=0}^{u-1}(-1)^{k}\left(\begin{array}{c}
u-1 \\
k
\end{array}\right)(s-1-m)_{u-1-k} \times \\
& \times(s-1-m)_{k}(t-1+n)_{k}(t-1-n)_{u-1-k} .
\end{aligned}
$$

В работе [7] ассоциативность Lone-Star-произведения только предполагалась, в настоящей работе она явно проверена. Чтобы получить ковариантное выражение из (48), нужно перемножить векторы старшего и младшего веса, в этом случае все свертки с $\epsilon_{\alpha \beta}$ ненулевые. После соответствующего переопределения индексов и подстановки $\nu=1-2 \lambda$ получим выражение (17). Затем нужно подставить его в условия ассоциативности (35)-(39). Но так как факториальный фактор из формулы (17)

$$
\frac{m ! n !}{(m-p) !(n-p) ! p !}
$$

дает все необходимые нули, т. е., например, $A(m, n-2,-1, \hat{\nu}) \equiv 0$, то можно рассматривать только уравнение $(37)$ при $p=1, \ldots, n$. Обозначим для краткости

$$
{ }_{4} F_{3}\left[\begin{array}{cccc}
1-\frac{\hat{\nu}}{2} & \frac{\hat{\nu}}{2} & \frac{-p}{2} & \frac{1-p}{2} ; 1 \\
\frac{1-m}{2} & \frac{1-n}{2} & \frac{m+n-2 p+3}{2} &
\end{array}\right]=F(m, n, p, \hat{\nu}) .
$$


Функция $F(m, n, p, \hat{\nu})$ должна удовлетворять тождеству

$$
\begin{aligned}
\frac{n !}{(n-p) ! p !} F(m, n, p, \hat{\nu})= & \frac{(n-2) !}{(n-p-2) ! p !} F(m, n-2, p, \hat{\nu})+ \\
& +2 \frac{(n-2) !}{(n-p-1) !(p-1) !} F(m, n-2, p-1, \hat{\nu})+ \\
& +\frac{(n-2) !}{(n-p) !(p-2) !} \frac{m+n-2 p+3-\hat{\nu}}{m+n-2 p+3} \times \\
& \times \frac{m+n-2 p+1+\hat{\nu}}{m+n-2 p+1} F(m, n-2, p-2, \hat{\nu}) .
\end{aligned}
$$

Лучше рассматривать гипергеометрические функции из правой части по отдельности.

Первое слагаемое $F(m, n-2, p, \hat{\nu})$. Согласно определению его можно представить в виде ряда

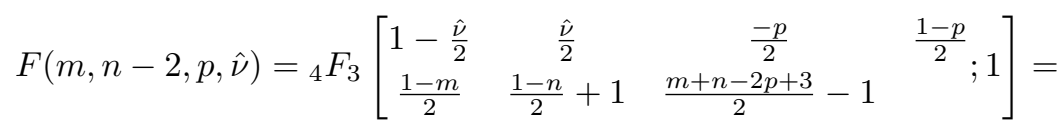

$$
\begin{aligned}
& =\sum_{q=0}^{\infty} \frac{\left(1-\frac{\hat{\nu}}{2}\right)_{q}\left(\frac{\hat{\nu}}{2}\right)_{q}\left(\frac{1-p}{2}\right)_{q}\left(-\frac{p}{2}\right)_{q}}{\left(\frac{1-m}{2}\right)_{q}\left(\frac{1-n}{2}+1\right)_{q}\left(\frac{m+n-2 p+3}{2}-1\right)_{q} q !} .
\end{aligned}
$$

Выполняя преобразование символов Похгаммера, получаем новое выражение для $F(m, n-2, p, \hat{\nu})$ :

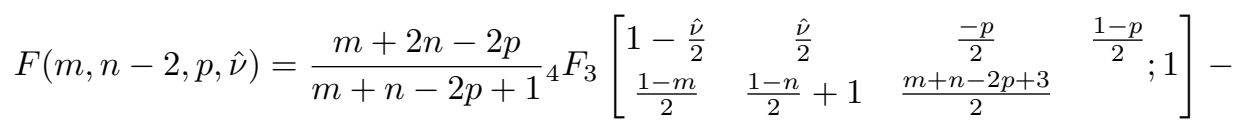

$$
\begin{aligned}
& -\frac{n-1}{m+n-2 p+1}{ }_{4} F_{3}\left[\begin{array}{cccc}
1-\frac{\hat{\nu}}{2} & \frac{\hat{\nu}}{2} & \frac{-p}{2} & \frac{1-p}{2} ; 1 \\
\frac{1-m}{2} & \frac{1-n}{2} & \frac{m+n-2 p+3}{2} &
\end{array}\right] \text {. }
\end{aligned}
$$

Второй член является гипергеометрической функцией из левой части условия ассоциативности (37).

Третье слагаемое $F(m, n-2, p-2, \hat{\nu})$. Здесь мы используем тот факт, что функция ${ }_{4} F_{3}(1)$ является заальшутсовской, и выполняем преобразование. Информацию об обрывающихся заальшутсовских рядах можно найти в монографии [13]. Пусть $p=2 N$ (нечетный случай может быть рассмотрен аналогично),

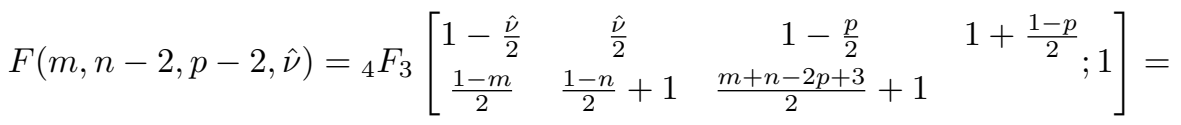

$$
\begin{aligned}
& =\frac{\left(\frac{p-m}{2}-1\right)_{N-1}}{\left(\frac{1-m}{2}\right)_{N-1}} \frac{\left(\frac{p-n}{2}\right)_{N-1}}{\left(\frac{1-n}{2}+1\right)_{N-1}} \times \\
& \times{ }_{4} F_{3}\left[\begin{array}{cccc}
1+\frac{m+n-2 p+1+\hat{\nu}}{2} & 1+\frac{m+n-2 p+3-\hat{\nu}}{2} & 1-\frac{p}{2} & \left.1+\frac{1-p}{2} ; 1\right] . \\
\frac{m}{2}-p+3 & \frac{n}{2}-p+2 & \frac{m+n-2 p+3}{2}+1 &
\end{array}\right] .
\end{aligned}
$$


Нужно выделить ряд, который совпадает с гипергеометрической функцией из левой части уравнения ассоциативности (37). После простых упражнений с переопределением индексов в сумме и преобразования символов Похгаммера (как в первом слагаемом) получаем новое выражение для третьего слагаемого:

$$
\begin{aligned}
& F(m, n-2, p-2, \hat{\nu})= \\
& =\frac{\left(\frac{p-m}{2}-1\right)_{N-1}}{\left(\frac{1-m}{2}\right)_{N-1}} \frac{\left(\frac{p-n}{2}\right)_{N-1}}{\left(\frac{1-n}{2}+1\right)_{N-1}} \frac{\left(\frac{m+n-2 p+3}{2}\right)\left(\frac{m}{2}-p+2\right)\left(\frac{n}{2}-p+1\right)}{\left(\frac{m+n-2 p+3-\hat{\nu}}{2}\right)\left(\frac{m+n-2 p+1+\hat{\nu}}{2}\right)\left(\frac{1-p}{2}\right)\left(\frac{-p}{2}\right)} \times \\
& \quad \times\left(\frac{m}{2}-p+1\right)\left({ }_{4} F_{3}\left[\begin{array}{cccc}
\frac{m+n-2 p+1+\hat{\nu}}{2} & \frac{m+n-2 p+3-\hat{\nu}}{2} & -\frac{p}{2} & \frac{1-p}{2} \\
\frac{m}{2}-p+1 & \frac{n}{2}-p+1 & \frac{m+n-2 p+3}{2}
\end{array}\right]-\right. \\
& \left.\quad-{ }_{4} F_{3}\left[\begin{array}{cccc}
\frac{m+n-2 p+1+\hat{\nu}}{2} & \frac{m+n-2 p+3-\hat{\nu}}{2} & -\frac{p}{2} & \frac{1-p}{2} ; 1 \\
\frac{m}{2}-p+2 & \frac{n}{2}-p+1 & \frac{m+n-2 p+3}{2} &
\end{array}\right]\right) .
\end{aligned}
$$

Второе слагаемое $F(m, n-2, p-1, \hat{\nu})$. Удобно рассмотреть второе слагаемое в конце. После преобразований первого и третьего слагаемых мы получили гипергеометрические функции из левой части (37) и некоторые дополнительные члены. Разделим второе слагаемое на 2

$$
\begin{aligned}
F(m, n-2, p-1, \hat{\nu}) & =A F(m, n-2, p-1, \hat{\nu})+B F(m, n-2, p-1, \hat{\nu}), \\
A+B & =1 .
\end{aligned}
$$

Слагаемое, содержащее множитель $A$, преобразуем как первую гипергеометрическую функцию, а слагаемое, содержащее множитель $B,-$ как третью. Константы $A$ и $B$ выбираются так, чтобы сократить дополнительные члены из первого и третьего слагаемых.

Преобразованное слагаемое, содержащее множитель $A$, имеет вид

$$
\begin{gathered}
F(m, n-2, p-1, \hat{\nu})=\frac{n-1}{p}{ }_{4} F_{3}\left[\begin{array}{cccc}
1-\frac{\hat{\nu}}{2} & \frac{\hat{\nu}}{2} & \frac{-p}{2} & \frac{1-p}{2} \\
\frac{1-m}{2} & \frac{1-n}{2} & \frac{m+n-2 p+3}{2} &
\end{array}\right]- \\
-\frac{(n-p-1)}{p}{ }_{4} F_{3}\left[\begin{array}{cccc}
1-\frac{\hat{\nu}}{2} & \frac{\hat{\nu}}{2} & \frac{-p}{2} & \frac{1-p}{2} ; 1 \\
\frac{1-m}{2} & \frac{1-n}{2}+1 & \frac{m+n-2 p+3}{2} &
\end{array}\right] .
\end{gathered}
$$

Преобразованное слагаемое, содержащее множитель $B$, имеет вид

$$
\begin{aligned}
& F(m, n-2, p-1, \hat{\nu})=\frac{\left(\frac{p-m}{2}\right)_{N}}{\left(\frac{1-m}{2}\right)_{N}} \frac{\left(\frac{p-n}{2}\right)_{N}}{\left(\frac{1-n}{2}\right)_{N}} \frac{n-1}{(n-p) p} \times
\end{aligned}
$$

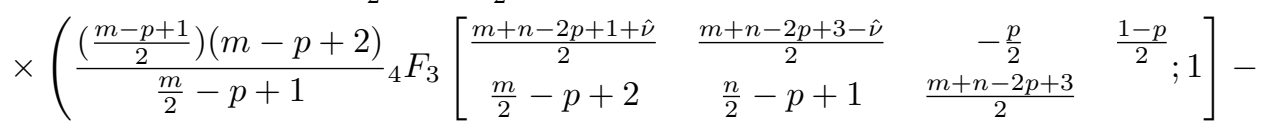

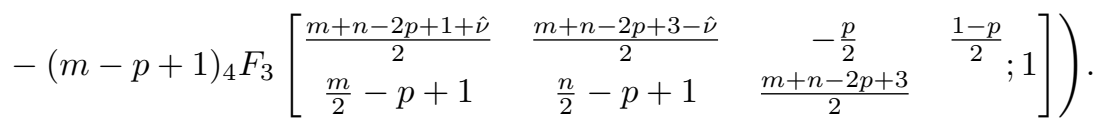

Значения $A$ и $B$, при которых дополнительные слагаемые сокращаются, следующие:

$$
A=\frac{m+2 n-2 p}{m+n-2 p+1}, \quad B=1-\frac{m+2 n-2 p}{m+n-2 p+1} .
$$

Подставляя все три слагаемых в условие ассоциативности (37), видим, что оно не нарушено. 
Благодарности. Автор благодарит М. А. Васильева за полезные комментарии.

\section{Список литературы}

[1] E. P. Wigner, "Do the equations of motion determine the quantum mechanical commutation relations?", Phys. Rev., 77:5 (1950), 711-712.

[2] M. A. Vasiliev, "Higher spin algebras and quantization on the sphere and hyperboloid", Internat. J. Modern Phys. A, 6:7 (1991), 1115-1135.

[3] М.А. Васильев, "Квантование на сфере и супералгебры высших спинов", Писъма в ЖКЭТФ, 50:8 (1989), 344-347.

[4] E. Bergshoeff, M. P. Blencowe, K. S. Stelle, "Area-preserving diffeomorphisms and higherspin algebras", Commun. Math. Phys., 128:2 (1990), 213-230.

[5] M. A. Vasiliev, "Consistent equation for interacting gauge fields of all spins in $(3+1)$-dimensions", Phys. Lett. B, 243:4 (1990), 378-382.

[6] M. A. Vasiliev, "Nonlinear equations for symmetric massless higher spin fields in $(A) d S_{d}$ ", Phys. Lett. B, 567:1 (2003), 139-151, arXiv: hep-th/0304049.

[7] C. N. Pope, X. Shen, L. J. Romans, " $W_{\infty}$ and the Racah-Wigner algebra", Nucl. Phys. B, 339:1 (1990), 191-221.

[8] E. Bergshoeff, M. A. Vasiliev, B. de Wit, "The super- $W_{\infty}(\lambda)$ algebra", Phys. Lett. B, 256:2 (1991), 199-205.

[9] E. Bergshoeff, B. de Wit, M. A. Vasiliev, "The structure of the super- $W_{\infty}(\lambda)$ algebra", Nucl. Phys. B, 366:2 (1991), 315-346.

[10] M. R. Gaberdiel, R. Gopakumar, "Minimal model holography", J. Phys. A: Math. Theor., 46:21 (2013), 214002, 47 pp., arXiv: 1207.6697.

[11] E. S. Fradkin, V. Ya. Linetsky, "Supersymmetric Racah basis, family of infinite-dimensional superalgebras, SU $(\infty+1 \mid \infty)$ and related 2D models", Modern Phys. Lett. A, 6:7 (1991), 617-633.

[12] ;po0E. Joung, K. Mkrtchyan, "Notes on higher-spin algebras: minimal representations and structure constants", JHEP, 05 (2014), 103, 34 pp., arXiv: 1401.7977.

[13] L. J. Slater, Generalized Hypergeometric Functions, Cambridge Univ. Press, Cambridge, 1966.

Поступила в редакцию 19.02.2016, после доработки 28.11.2016 